\title{
New aspects of poor nutrition in the life cycle within the fractional calculus
}

\author{
Dumitru Baleanu ${ }^{1,2}$, Amin Jajarmi $^{3^{*}}$, Ebenezer Bonyah ${ }^{4}$ and Mojtaba Hajipour ${ }^{5}$
}

\author{
"Correspondence: a.jajarmi@ub.ac.ir \\ ${ }^{3}$ Department of Electrical \\ Engineering, University of Bojnord, \\ Bojnord, Iran \\ Full list of author information is \\ available at the end of the article
}

\begin{abstract}
The nutrition of pregnant women is crucial for giving birth to a healthy baby and even for the health status of a nursing mother. In this paper, the poor nutrition in the life cycle of humans is explored in the fractional sense. The proposed model is examined via the Caputo fractional operator and a new one with Mittag-Leffler (ML) nonsingular kernel. The stability analysis as well as the existence and uniqueness of the solution are investigated, and an efficient numerical scheme is also designed for the approximate solution. Comparative numerical analysis of these two operators reveals that the model based on the new fractional derivative with ML kernel has a different asymptotic behavior to the classic Caputo. Thus, the new aspects of fractional calculus provide more flexible models which help us to adjust the dynamical behaviors of the real-world phenomena better.
\end{abstract}

Keywords: Malnutrition; Fractional calculus; Caputo; Mittag-Leffler function

\section{Introduction}

Nutrition has been a major factor in every human stage of development from conception to old age. The quality of nutrition determines the quality of life and particularly the health of a pregnant woman [1]. When a pregnant woman has a poor nutritional status, then the fetus in the womb begins to encounter many life challenges beginning from birth to adulthood. The quality of health care of a pregnant woman is crucial to the quality of health of the child to be born [2]. In other words, the nutrient gain or loss has an effect on the weight of the child. If a newborn child has a weight of less than $2.5 \mathrm{~kg}$, it is deemed to be low birth weight. This situation is characterized by infant mortality, poor growth, poor mental development, damage in brain, anaemia, low body, etc. [3, 4].

Sometimes the newborn babies are put into incubators for some number of days and monitored. The quality of human life cycle depends on the quality of nutrients consumed by the pregnant woman during this stage. In the developing countries, such as Sub-Saharan Africa, the situation is worse; many families find it difficult to feed pregnant women with quality food containing the required nutrients. In some cases, due to religious and cultural believes, many pregnant women are denied of a nutritious diet [5-7].

An undergrown child, which is also a result of poor nutrition during pregnancy, is viewed as a child whose weight or associated height is totally different from what he/she ought to be at that age. There are other factors that usually account for this situation, including heredity, malnutrition, and improper balanced diet $[3,5,8]$. In fact, this under-

(c) The Author(s) 2018. This article is distributed under the terms of the Creative Commons Attribution 4.0 International License (http://creativecommons.org/licenses/by/4.0/), which permits unrestricted use, distribution, and reproduction in any medium, provided you give appropriate credit to the original author(s) and the source, provide a link to the Creative Commons license, and indicate if changes were made. 
growth becomes a serious issue at the adolescent age of the child [9]. There are a number of interventions designed to mitigate this effect of undergrowth at the adolescent stage. For instance, UNICEF tries to improve the quality of nutrition of adolescent girls because they are potentially women capable of giving birth $[9,10]$. It has been established that the first year of adolescent life of a woman requires more energy and nutrients, and UNICEF provides supplements such as iron and folate through workshops during this stage of development [11-13]. The life cycle which is the bedrock of human race calls for the need to invest in the provision of adequate nutrients to humans, particularly pregnant women [14].

Mathematical modeling has become a vital tool to investigate many societal problems and provides cost-effective mechanisms in solving such problems. There are several mathematical models on the epidemiology of diseases $[15,16]$; however, there is a scanty information on the mathematical modeling of a life cycle. All the studies carried out on malnutrition have been focused on the local differentiation without any cogent justification. It is worthy to note that the local differentiation has fallen short to live up to the expectation because of the nonlocality effect into mathematical formation. Therefore, there is a need to formulate mathematical problems that describe the nonlocality of biological systems. Among the existing approaches, the fractional calculus (FC) has a singular and unique characteristic of capturing memory effects, which is found in almost all biological processes.

The FC has played a significant role in modeling different kinds of diseases [17-21]. There are several fractional operators, such as Caputo and Caputo-Fabrizio, which have been applied to study the behavior of biological systems. Regarding these operators, some efficient numerical techniques have also been developed to solve different types of fractional differential equations [22-25]. However, a few attempts have been made to compare these operators in order to determine the most efficient one. In [26] the computer virus dynamics have been studied via the Caputo and Beta derivatives. In [27] the authors proposed mathematical model in the fractional sense on immunogenetic tumor and observed that tumor growth rate, source rate of immune cells, and death rate of immune cells constitute a major factor in tumor dynamics system. Also, the authors in [28] proposed a mathematical model of hepatitis $\mathrm{C}$ using the Caputo-Fabrizio derivative to investigate the dynamic of the disease. In [29] the authors proposed a fractional mathematical malaria model to investigate the transmission dynamics with control strategies. The fractional complex-order model for HIV infection with drug resistance during therapy was also studied in [30] to examine the dynamic of HIV. In [31] the authors used a fractional order HIV model to explore the effect of vaccines on backward bifurcation. In [32], a new fractional epidemic model with vaccination was introduced by using the Caputo fractional derivative. Some other noticeable efforts have also been made in [33, 34].

The mathematical modeling of realistic systems with memory effects has been a big challenge for many scientists, since the nonlocal dynamics cannot always be properly characterized by the classic version of fractional derivatives (FDs) with singular kernel. Hence, to describe the nonlocality of complex processes better, some new definitions of the FDs with nonlocal and nonsingular kernel have been introduced into the literature. Among the existing definitions, a new FD with Mittag-Leffler (ML) kernel is one of the most desirable candidates to model the real-world problems [35]. Indeed, this new fractional differentiation is more natural and more suitable to model realistic systems than the other FC 
derivatives due to the wide applicability of ML function as well as its nonlocal and nonsingular characteristics. Applying this new definition, a different type of nonlocal dynamics is exhibited in comparison with the classical fractional dynamics. Indeed, the asymptotic behaviors within this derivative are different from those of the classical FC differentiations. However, the advantages of models within this new derivative should be deeply examined compared to the other FC derivatives, and efficient numerical methods to solve the fractional differential equations within this calculus should be continuously investigated. Inspired by the above discussions, this paper aims to develop a new fractional mathematical model to investigate new aspects of the poor nutrient status of pregnant women. In this formulation, we use the new definition of FDs with ML nonsingular kernel [35]. We also provide an efficient numerical scheme to solve the fractional model under consideration. Numerical simulations verify that there exists a new asymptotic behavior within this new formulation, which is different from that exhibited by using the other FC derivatives. Hence, this new model has the potential to better represent hidden aspects of the poor nutrition system in the life cycle than the classic type of fractional operators.

The paper is arranged as follows. First, the mathematical basic definitions and notations are given. Then, we present the mathematical formulation of poor nutrition of pregnant women in a fractional sense. After that, we investigate the stability analysis of the proposed model. Next, the existence and uniqueness of the solution related the fractional poor nutrition system are given. In the next section, we develop a numerical scheme to solve the fractional model under consideration. Finally, we present our numerical findings, which are compared with those obtained within the classic Caputo FD.

\section{Basic definitions and preliminaries}

This section briefly gives some preliminaries concerning the FDs. There are some definitions for the FDs including Riemann-Liouville, Weyl, Caputo, Marchaud, and Riesz [36-38]. Moreover, a new definition of FDs with ML kernel has been developed to model some realistic systems [35]. Starting with the classic Caputo, we present the following definitions.

Definition 1 For a given function $x:[a, b] \rightarrow \mathbb{R}$, the (left) Caputo FD of order $\alpha$ is given as

$$
{ }_{a}^{C} D_{t}^{\alpha} x(t)=\frac{1}{\Gamma(n-\alpha)} \int_{a}^{t} x^{(n)}(\xi)(t-\xi)^{n-\alpha-1} d \xi, \quad n-1<\alpha \leq n .
$$

Also, the associated Caputo fractional integral is defined by

$$
{ }_{a}^{C} I_{t}^{\alpha} x(t)=\frac{1}{\Gamma(\alpha)} \int_{a}^{t} x(\xi)(t-\xi)^{\alpha-1} d \xi
$$

The characteristic principles underpinning the Caputo derivative and integral can be found in [36-38].

Definition 2 ([35]) Let $x \in H^{1}(a, b), b>a$, and $0<\alpha<1$. Then, the new FD of $x$ in the Caputo sense denoted by the $\mathrm{ABC}$ is defined by

$$
{ }_{a}^{\mathrm{ABC}} D_{t}^{\alpha} x(t)=\frac{B(\alpha)}{1-\alpha} \int_{a}^{t} x^{\prime}(\xi) E_{\alpha}\left[-\frac{\alpha}{1-\alpha}(t-\xi)^{\alpha}\right] d \xi
$$


in which $B(\alpha)$ denotes the normalization function satisfying $B(0)=B(1)=1$ and $E_{\alpha}$ is the ML function

$$
E_{\alpha}(z)=\sum_{k=0}^{\infty} \frac{\left(z^{\alpha}\right)^{k}}{\Gamma(\alpha k+1)}, \quad \alpha>0 .
$$

Also, the associated $\mathrm{ABC}$ fractional integral is given as

$$
{ }_{a}^{\mathrm{ABC}} I_{t}^{\alpha} x(t)=\frac{1-\alpha}{B(\alpha)} x(t)+\frac{\alpha}{B(\alpha) \Gamma(\alpha)} \int_{a}^{t} x(\xi)(t-\xi)^{\alpha-1} d \xi .
$$

The new definition presented above is crucial when dealing with the real life problems and plays an important rule when considering a problem of Laplace transform with the initial condition. For more details, the interested reader can refer to [39-43].

\section{Mathematical model formulation}

This section presents a mathematical formulation of malnutrition in pregnant females. The dynamic of this phenomenon has been shown in Fig. 1. The undernourished pregnant females $\left(S_{f}\right)$ give birth to malnourished boys $\left(M_{b}\right)$ and girls $\left(M_{g}\right)$. The boys and girls are malnourished from susceptible females at the transmission rates $\beta_{b}$ and $\beta_{g}$, respectively. When the low weight babies are not given the proper medical attention, they turn to become undergrown children at the rates of $\alpha_{b}$ and $\alpha_{g}$ for boys and girls, respectively. The natural mortality rate is denoted by $\mu$, and the number of underweight individuals is $U$. The total population is denoted by $N_{h}$, where $N_{h}=S_{f}+M_{b}+M_{g}+U$. The following system of nonlinear differential equations describes the poor nutrition at different stages of a life cycle [44]:

$$
\left\{\begin{array}{l}
\frac{d S_{f}}{d t}=(B+\varepsilon)-\left(\beta_{b} M_{b}+\beta_{g} M_{g}+\mu\right) S_{f}+\eta_{g} M_{g} \\
\frac{d M_{b}}{d t}=\beta_{b} S_{f} M_{b}-\left(\mu_{b}+\alpha_{b}+\mu\right) M_{b}+\gamma_{b} U \\
\frac{d M_{g}}{d t}=\beta_{g} S_{f} M_{g}-\left(\alpha_{g}+\eta_{g}+\mu\right) M_{g}+\gamma_{g} U \\
\frac{d U}{d t}=\alpha_{b} M_{b}+\alpha_{g} M_{g}-\left(\gamma_{b}+\gamma_{g}+\mu\right) U
\end{array}\right.
$$

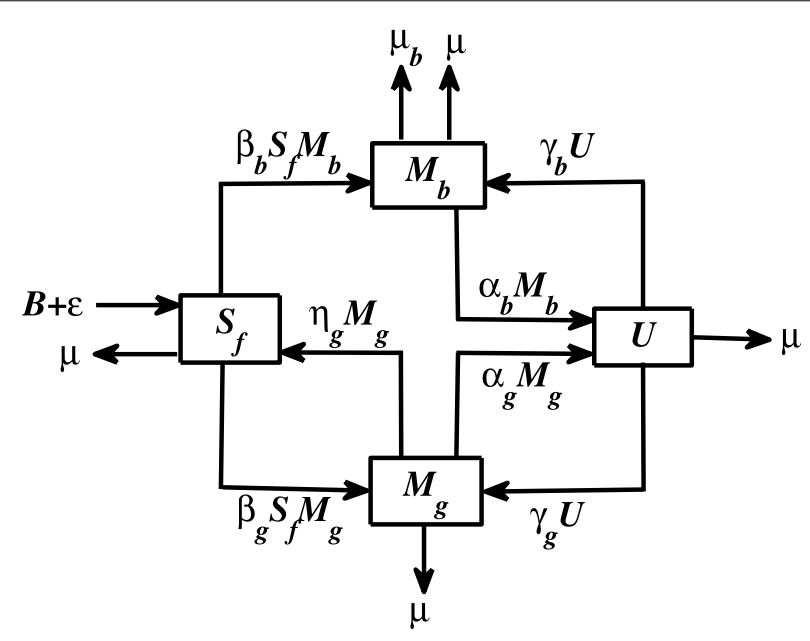

Figure 1 The transmission of malnutrition and underweight 
where $B$ is the new female recruitment rate and $\varepsilon$ denotes the vertical transmission to a new born population. The other parameters $\eta_{g}, \gamma_{b}, \gamma_{g}$ in Eq. (6) also represent the recovery rate of malnourished girls and the fractions of underweight babies moving to $M_{b}$ and $M_{g}$, respectively. Since model (6) concerns the human population dynamics, the entire parameters and state variables are considered to be positive and nonnegative, respectively [44-46].

The mathematical model given by Eq. (6) has been previously examined in [44] to study the transmission of malnutrition and underweight individuals in the society. However, this model does not include the effects of memory, which are found in many biological systems. Hence, in order to take into account the memory effects by the mathematical formulation, we modify the model by replacing the ordinary derivative to the newly introduced $A B C$ fractional operator. For this purpose, let us obtain the fractional representation of Eq. (6) by considering the operator ${ }_{0}^{\mathrm{ABC}} D_{t}^{\alpha}$ for each derivative. Then the transmission of poor nutrition system (6) in the fractional $\mathrm{ABC}$ sense is given as follows:

$$
\left\{\begin{array}{l}
{ }_{0}^{\mathrm{ABC}} D_{t}^{\alpha} S_{f}=(B+\varepsilon)-\left(\beta_{b} M_{b}+\beta_{g} M_{g}+\mu\right) S_{f}+\eta_{g} M_{g} \\
{ }_{0}^{\mathrm{ABC}} D_{t}^{\alpha} M_{b}=\beta_{b} S_{f} M_{b}-\left(\mu_{b}+\alpha_{b}+\mu\right) M_{b}+\gamma_{b} U, \\
{ }_{0}^{\mathrm{ABC}} D_{t}^{\alpha} M_{g}=\beta_{g} S_{f} M_{g}-\left(\alpha_{g}+\eta_{g}+\mu\right) M_{g}+\gamma_{g} U \\
{ }_{0}^{\mathrm{ABC}} D_{t}^{\alpha} U=\alpha_{b} M_{b}+\alpha_{g} M_{g}-\left(\gamma_{b}+\gamma_{g}+\mu\right) U .
\end{array}\right.
$$

\section{Stability analysis}

In this section, we shall endeavor to analyze the model stability.

Lemma 1 The closed set

$$
\Psi=\left\{\left(S_{f}, M_{b}, M_{g}, U\right) \in \mathbb{R}_{+}^{4}: S_{f}+M_{b}+M_{g}+U \leq \frac{B+\varepsilon}{\mu}\right\}
$$

is positively invariant with respect to the system given by Eq. (7).

Proof For system (7), the FD of the total population is expressed as

$$
{ }_{0}^{\mathrm{ABC}} D_{t}^{\alpha} N_{h}=B+\varepsilon-\mu N_{h}(t)-\mu_{b} M_{b} \leq B+\varepsilon-\mu N_{h}(t)
$$

since $\mu_{b}>0$ and $M_{b} \geq 0$. Using the Laplace transform, from Eq. (9) we derive

$$
\begin{aligned}
N_{h}(t) \leq & \left(\frac{B(\alpha)}{B(\alpha)+(1-\alpha) \mu} N_{h}(0)+\frac{(1-\alpha)(B+\varepsilon)}{B(\alpha)+(1-\alpha) \mu}\right) E_{\alpha, 1}\left(-\beta t^{\alpha}\right) \\
& +\frac{\alpha(B+\varepsilon)}{B(\alpha)+(1-\alpha) \mu} E_{\alpha, \alpha+1}\left(-\beta t^{\alpha}\right)
\end{aligned}
$$

where $\beta=\frac{\alpha \mu}{B(\alpha)+(1-\alpha) \mu}$ and $E_{\alpha, \beta}$ is the two-parameter ML function. By taking into account that the ML function possesses an asymptotic characteristic

$$
E_{\alpha_{1}, \alpha_{2}}(\xi) \sim-\sum_{k}^{\infty} \frac{\xi^{-k}}{\Gamma\left(\alpha_{2}-\alpha_{1} k\right)}+o\left(|\xi|^{-1-\varpi}\right) \quad\left(|\xi| \rightarrow \infty, \frac{\alpha_{1} \pi}{2}<|\arg (\xi)| \leq \pi\right),
$$


it is obvious to see that $N_{h}(t) \leq \frac{B+\varepsilon}{\mu}$ as $t \rightarrow \infty$. Thus, the entire solutions of the proposed system for the initial conditions belonging to $\Psi$ remain in $\Psi$ for every $t>0$. Hence, $\Psi$ is a positively invariant region with respect to system (7).

The system given by Eq. (7) has three equilibrium points $E_{0}, E_{1}, E_{2}$ for the set of parameters given in [44]. However, among these three points, only the first one, i.e., $E_{0}$, satisfies the system requirement limitations $S_{f} \geq 0, M_{b} \geq 0, M_{g} \geq 0, U \geq 0$, and the other ones $E_{1}, E_{2}$ have no physical interpretation in our case. Hence, within this manuscript, we discuss only the equilibrium point $E_{0}$ of system (7), viz. $E_{0}=\left(\frac{B+\varepsilon}{\mu}, 0,0,0,0\right)$. Indeed, $E_{0}$ is the malnutrition-free equilibrium of pregnant women. The Jacobian matrix $J_{E_{0}}$ of (7) evaluated at the disease-free equilibrium $E_{0}$ is given by

$$
J_{E_{0}}=\left(\begin{array}{cccc}
-\mu & -\frac{\beta_{b}(B+\varepsilon)}{\mu} & -\frac{\beta_{g}(B+\varepsilon)}{\mu}+\eta_{g} & 0 \\
0 & \frac{\beta_{b}(B+\varepsilon)}{\mu}-\mu_{b}-\alpha_{b}-\mu & 0 & \gamma_{b} \\
0 & 0 & \frac{\beta_{g}(B+\varepsilon)}{\mu}-\eta_{g}-\alpha_{g}-\mu & \gamma_{g} \\
0 & \alpha_{b} & \alpha_{g} & -\left(\gamma_{b}+\gamma_{g}+\mu\right)
\end{array}\right)
$$

Also, from Theorem 2 of [47] the basic reproduction number of system (7) is given as

$$
R_{0}=\frac{\beta_{g}(B+\varepsilon)\left(\left(\mu_{b}+\mu\right)\left(\mu+\gamma_{b}+\gamma_{g}\right)+\alpha_{b}\left(\mu+\gamma_{g}\right)\right)}{\mu\left(\mu+\mu_{b}\right)\left(\mu\left(\mu+\alpha_{g}+\eta_{g}+\gamma_{b}+\gamma_{g}\right)+\gamma_{b}\left(\alpha_{g}+\eta_{g}\right)+\gamma_{g} \eta_{g}\right)} .
$$

Note that the malnutrition-free equilibrium $E_{0}$ of system (7) is asymptotically stable if $R_{0}<1$ [47].

\section{The existence and uniqueness of the solution}

In this section, we study the existence and uniqueness of the solution associated with the fractional-order poor nutrition model (7). To this end, first we reformulate the fractional poor nutrition system (7) in the form

$$
\left\{\begin{array}{l}
{ }_{0}^{\mathrm{ABC}} D_{t}^{\alpha} x(t)=f(x(t)), \quad 0<t<T<\infty, \\
x(0)=x_{0}
\end{array}\right.
$$

where $x$ is the state vector given by $x=\left(S_{f}, M_{b}, M_{g}, U\right), f$ is a real-valued continuous vector function defined by

$$
f=\left[\begin{array}{c}
f_{1} \\
f_{2} \\
f_{3} \\
f_{4}
\end{array}\right]=\left[\begin{array}{c}
(B+\varepsilon)-\left(\beta_{b} M_{b}+\beta_{g} M_{g}+\mu\right) S_{f}+\eta_{g} M_{g} \\
\beta_{b} S_{f} M_{b}-\left(\mu_{b}+\alpha_{b}+\mu\right) M_{b}+\gamma_{b} U \\
\beta_{g} S_{f} M_{g}-\left(\alpha_{g}+\eta_{g}+\mu\right) M_{g}+\gamma_{g} U \\
\alpha_{b} M_{b}+\alpha_{g} M_{g}-\left(\gamma_{b}+\gamma_{g}+\mu\right) U
\end{array}\right]
$$

and $x_{0}$ is the initial state vector. Since $f$ is a quadratic vector function, it fulfills the Lipschitz condition, i.e., there exists a constant $M$ such that

$$
\|f(x(t))-f(y(t))\| \leq M\|x(t)-y(t)\| .
$$


Note that the existence and uniqueness of the solution of fractional differential equation (14) in the sense of classic Caputo have been developed and analyzed in [36]. In the following theorem, we will investigate this subject theoretically for the $\mathrm{ABC}$ fractional operator with ML nonsingular kernel.

Theorem 1 (Existence and uniqueness) The fractional poor nutrition system (14) has a unique solution if the following condition holds:

$$
\frac{1-\alpha}{B(\alpha)} M+\frac{\alpha}{B(\alpha) \Gamma(\alpha)} M T^{\alpha}<1
$$

Proof See the Appendix.

\section{Numerical method}

In this section, we develop an efficient approximation scheme, namely the predictorcorrector method, for the numerical solution of fractional differential equation (14). Note that this technique has been previously investigated in [23-25] for different types of fractional differential equations. To develop this method for Eq. (14) in the sense of ABC FD, first we apply the $\mathrm{ABC}$ fractional integration of order $\alpha$ which provides Eq. (28). Consider a uniform mesh on $[0, T]$ and label the nodes $0,1, \ldots, N$, where $N$ is an arbitrary positive integer and $h_{N}=\frac{T-0}{N}$ is the time step size. We denote by $x_{i}$ the numerical approximation of $x\left(t_{i}\right)$. Then from Eq. (28) we obtain

$$
x_{i+1}=x_{0}+\frac{1-\alpha}{B(\alpha)} f\left(x_{i+1}\right)+\frac{\alpha}{B(\alpha) \Gamma(\alpha)} \int_{0}^{t_{i+1}}\left(t_{i+1}-\xi\right)^{\alpha-1} f(x(\xi)) d \xi
$$

Using the trapezoidal quadrature rule for the integration part in (18), we have

$$
\int_{0}^{t_{i+1}}\left(t_{i+1}-\xi\right)^{\alpha-1} f(x(\xi)) d \xi \approx \int_{0}^{t_{i+1}}\left(t_{i+1}-\xi\right)^{\alpha-1} \tilde{f}_{i+1}(\xi) d \xi
$$

where $\tilde{f}_{i+1}(\xi)$ is the following piecewise interpolation polynomial with degree one:

$$
\left.\tilde{f}_{i+1}(\xi)\right|_{\xi \in\left[t_{j}, t_{j+1}\right]} \approx \frac{t_{j+1}-\xi}{t_{j+1}-t_{j}} f\left(x_{j}\right)+\frac{\xi-t_{j}}{t_{j+1}-t_{j}} f\left(x_{j+1}\right), \quad 0 \leq j \leq i
$$

Inserting the above $\tilde{f}_{i+1}(\xi)$ into Eq. (19), we get the product trapezoidal quadrature formula

$$
\int_{0}^{t_{i+1}}\left(t_{i+1}-\xi\right)^{\alpha-1} \tilde{f}_{i+1}(\xi) d \xi=\frac{h_{N}^{\alpha}}{\alpha(\alpha+1)} \sum_{j=0}^{i+1} a_{j, i+1} f\left(x_{j}\right)
$$

where

$$
a_{j, i+1}= \begin{cases}i^{\alpha+1}-(i-\alpha)(i+1)^{\alpha}, & j=0 \\ (i-j+2)^{\alpha+1}-2(i-j+1)^{\alpha+1}+(i-j)^{\alpha+1}, & 1 \leq j \leq i \\ 1, & j=i+1\end{cases}
$$


As a consequence, the corrector formula is

$$
x_{i+1}=x_{0}+\frac{1-\alpha}{B(\alpha)} f\left(x_{i+1}^{p}\right)+\frac{\alpha h_{N}^{\alpha}}{B(\alpha) \Gamma(\alpha+2)}\left(a_{i+1, i+1} f\left(x_{i+1}^{p}\right)+\sum_{j=0}^{i} a_{j, i+1} f\left(x_{j}\right)\right)
$$

In addition, the predictor formula is obtained by using the product rectangle rule

$$
\int_{0}^{t_{i+1}}\left(t_{i+1}-\xi\right)^{\alpha-1} \tilde{f}(x(\xi)) d \xi \approx \frac{h_{N}^{\alpha}}{\alpha} \sum_{j=0}^{i} b_{j, i+1} f\left(x_{j}\right)
$$

where

$$
b_{j, i+1}=(i-j+1)^{\alpha}-(i-j)^{\alpha}, \quad 0 \leq j \leq i .
$$

Hence, the predictor formula is derived

$$
x_{i+1}^{p}=x_{0}+\frac{1-\alpha}{B(\alpha)} f\left(x_{i}\right)+\frac{h_{N}^{\alpha}}{B(\alpha) \Gamma(\alpha)} \sum_{j=0}^{i} b_{j, i+1} f\left(x_{j}\right) .
$$

Similar to the predictor-corrector scheme for the ODEs, we first use Eq. (26) to get $x_{i+1}^{p}$ (predictor), then we use (23) to get $x_{i+1}$ (corrector).

\section{Simulation results}

For the numerical simulation, we use the predictor-corrector scheme developed in the previous section. Figure 2 shows the simulation curves of $S_{f}, M_{b}, M_{g}$, and $U$ considering different values of $\alpha$, where the system parameters and initial conditions are selected as in
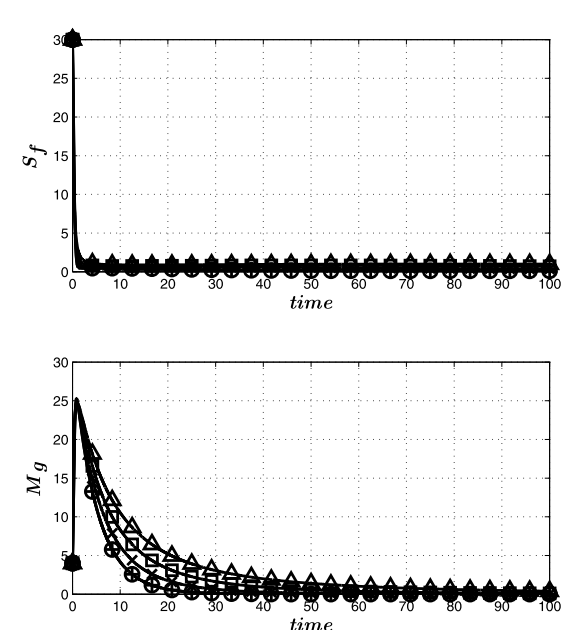
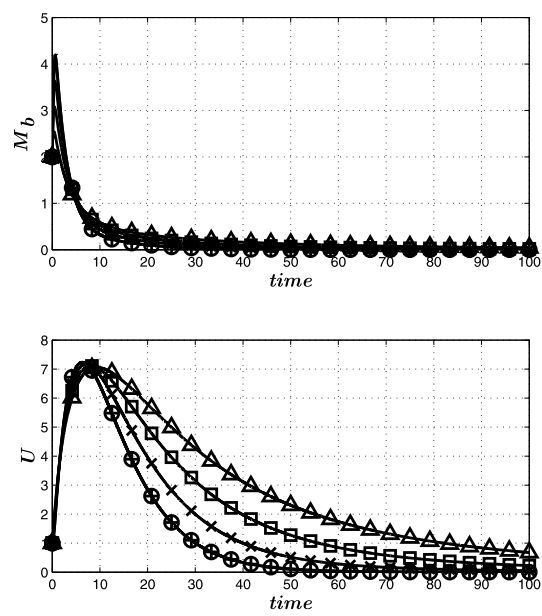

Figure 2 Simulation curves of $S_{f}, M_{b}, M_{g}$ and $U$ for different values of $\alpha . \alpha=0.85(\triangle), \alpha=0.9(\square), \alpha=0.95$ $(\times), \alpha=1(\bigcirc)$ and classic integer $(+)$ 

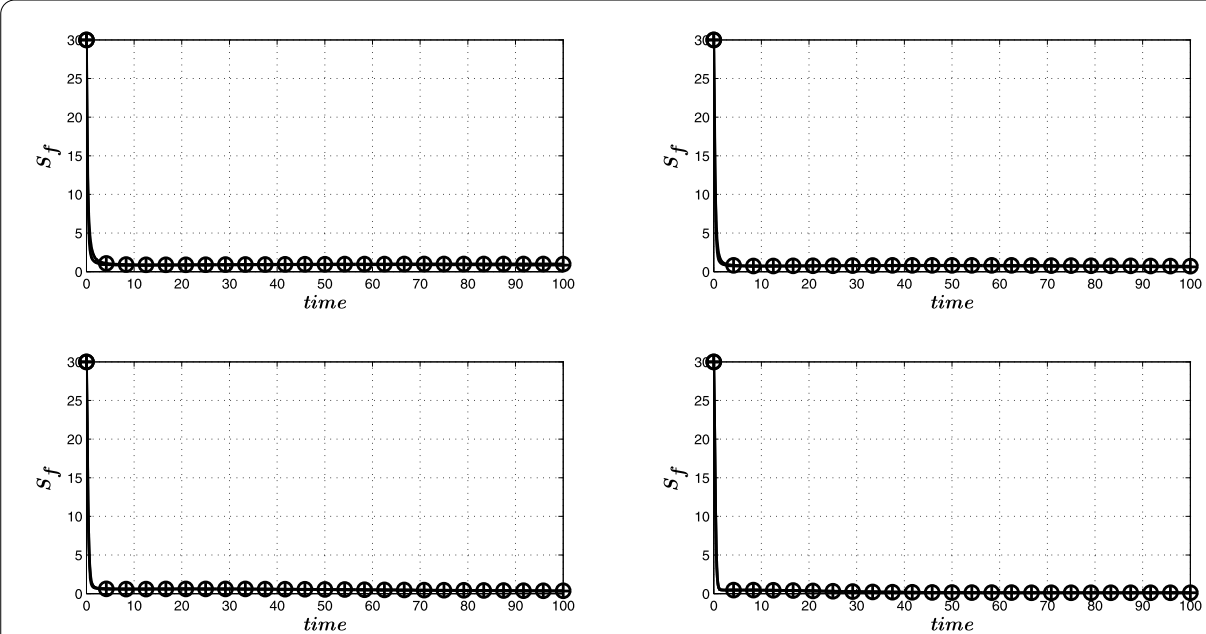

Figure 3 Asymptotic behaviour of $S_{f}$ within the classic Caputo derivative $(+)$ and $A B C$ fractional operator $(O)$ for different values of $\alpha . \alpha=0.85$ (upper-left), $\alpha=0.9$ (upper-right), $\alpha=0.95$ (lower-left), $\alpha=1$ (lower-right)
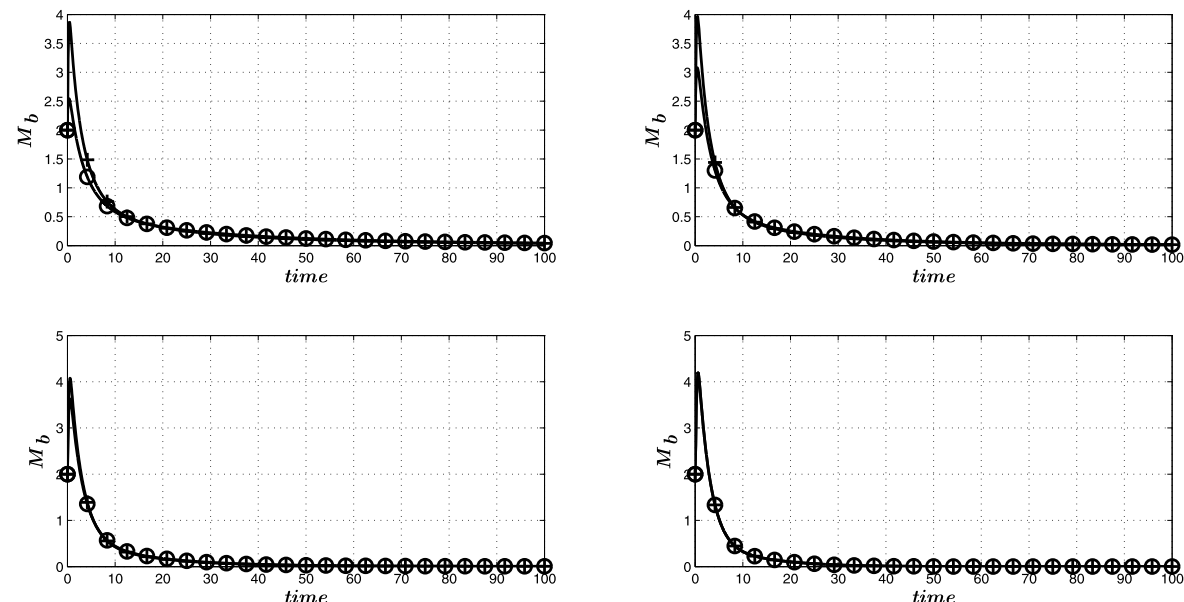

Figure 4 Asymptotic behaviour of $M_{b}$ within the classic Caputo derivative (+) and $A B C$ fractional operator (○) for different values of $\alpha . \alpha=0.85$ (upper-left), $\alpha=0.9$ (upper-right), $\alpha=0.95$ (lower-left), $\alpha=1$ (lower-right)

[44] as follows:

$$
\begin{array}{lll}
B=0.01, & \varepsilon=0.001, & \beta_{b}=0.1, \quad \beta_{g}=0.2, \\
\alpha_{b}=0.01, & \alpha_{g}=0.1, & \mu=0.1, \quad \mu_{b}=0.3, \\
\eta_{g}=0.1, & \gamma_{b}=0.01, & \gamma_{g}=0.01, \\
S_{f}(0)=30, \quad M_{b}(0)=2, & M_{g}(0)=4, \quad U(0)=1 .
\end{array}
$$

In this figure, we also provide the numerical solution of classic integer model (6) by using the fourth-order Runge-Kutta (RK) method. The results given in this figure indicate that the dynamical behavior of the poor nutrition model depends notably on the FD or$\operatorname{der} \alpha$. Indeed, the fractional poor nutrition system (7) within the ABC fractional operator 

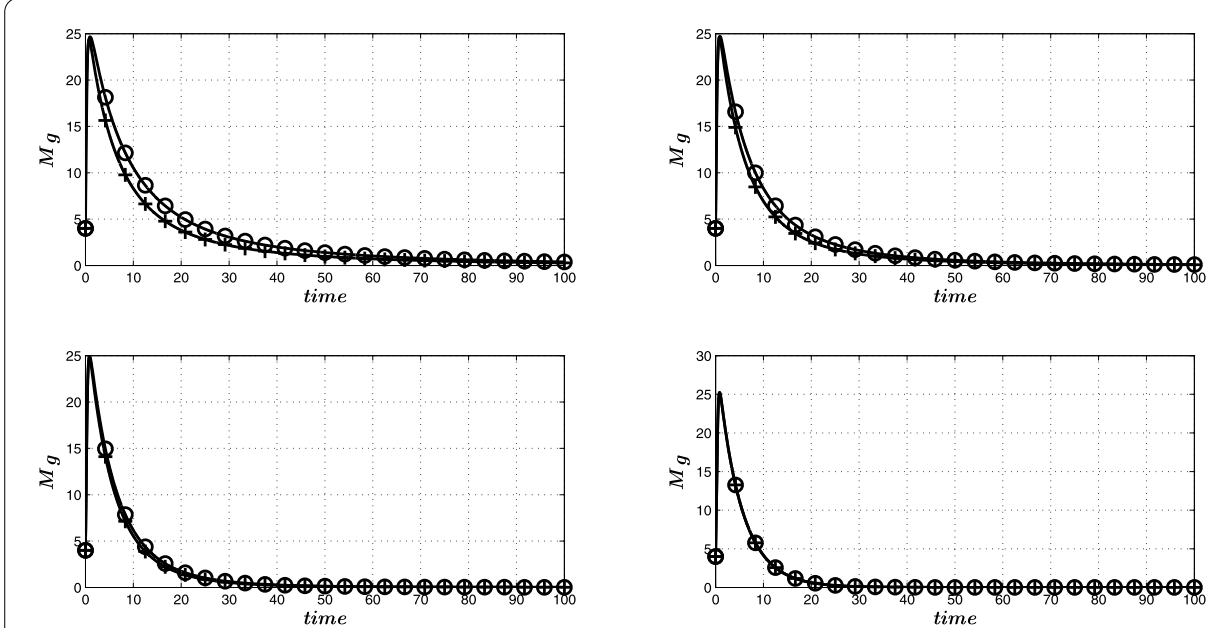

Figure 5 Asymptotic behaviour of $M_{g}$ within the classic Caputo derivative $(+)$ and $A B C$ fractional operator (O) for different values of $\alpha . \alpha=0.85$ (upper-left), $\alpha=0.9$ (upper-right), $\alpha=0.95$ (lower-left), $\alpha=1$ (lower-right)
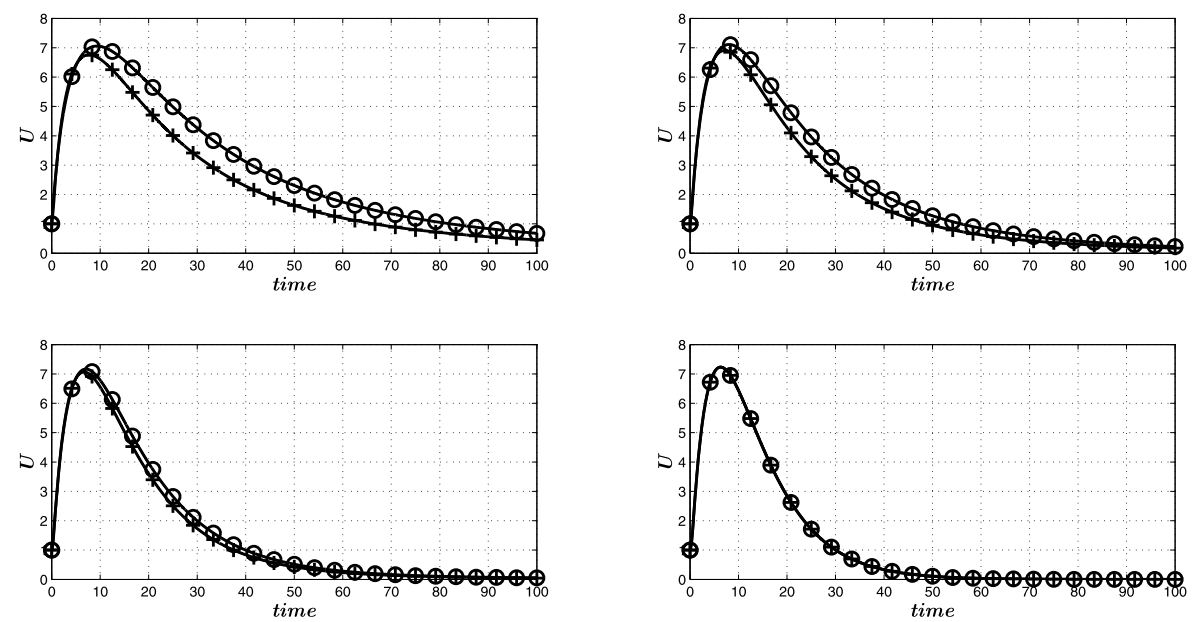

Figure 6 Asymptotic behaviour of $U$ within the classic Caputo derivative $(+)$ and $A B C$ fractional operator $(O)$ for different values of $\alpha . \alpha=0.85$ (upper-left), $\alpha=0.9$ (upper-right), $\alpha=0.95$ (lower-left), $\alpha=1$ (lower-right)

exhibits a new asymptotic behavior due to memory effects, which was invisible when modeling at $\alpha=1$. In addition, the numerical solution of Eq. (7) in fractional sense tends to the classic integer solution, as $\alpha$ tends to 1 . In Figs. 3, 4, 5, 6 we provide the simulation curves by taking into account two different fractional operators including the classic Caputo and the $\mathrm{ABC}$. As it is shown in these figures, there exist significant differences between the asymptotic behavior of fractional poor nutrition system within these two fractional operators. Comparative numerical analysis in Figs. 3, 4, 5, 6 indicates that the model based on the new FD with ML nonsingular kernel exhibits different asymptotic behavior to the classic Caputo. Thus, the recent prospects of the FC supply more flexible models to extract hidden features of the real-world phenomena. 


\section{Conclusion}

Mathematical modeling plays a critical role in investigating social problems and provides cost effective mechanisms to solve them. Among the existing approaches, the FC has a special characteristic of capturing memory effects, which is found in almost all biological systems. In this paper, we considered a fractional model of pregnant females by using the $\mathrm{ABC}$ fractional operator. The stability analysis and the existence and uniqueness of the solution for the considered model were studied. A predictor-corrector method was also developed for the numerical solution of fractional poor nutrition system. Numerical results reported in this manuscript confirmed that the proposed method is effective to solve the fractional dynamic system (7) for different values of $\alpha$. In addition, as it is interpreted from Fig. 2, the numerical solution in fractional sense approaches the classic case, as $\alpha$ approaches 1. Figures Figs. 3, 4, 5, 6 also indicated that the model based on the new ABC FD presents different asymptotic behavior to the classic Caputo. Thus, the recent prospects of FC supply more flexible models to extract hidden features of the real-world phenomena. In the future works, the most reliable fractional model can be obtained by selecting a relevant fractional operator in accordance with real data. Then, the administration of drugs or treatment can be recommended to each individual patient by using the information from the generalized model within the most relevant fractional operator.

\section{Appendix: The proof of Theorem 1}

Applying the $\mathrm{ABC}$ fractional integral operator (5), we obtain

$$
x(t)=x_{0}+\frac{1-\alpha}{B(\alpha)} f(x(t))+\frac{\alpha}{B(\alpha) \Gamma(\alpha)} \int_{0}^{t}(t-\xi)^{\alpha-1} f(x(\xi)) d \xi .
$$

Let $J=(0, T)$ and define the operator $F: \mathcal{C}\left(J, \mathbb{R}^{5}\right) \rightarrow \mathcal{C}\left(J, \mathbb{R}^{5}\right)$ as

$$
F[x(t)]=x_{0}+\frac{1-\alpha}{B(\alpha)} f(x(t))+\frac{\alpha}{B(\alpha) \Gamma(\alpha)} \int_{0}^{t}(t-\xi)^{\alpha-1} f(x(\xi)) d \xi
$$

Then Eq. (28) is reformulated as follows:

$$
x(t)=F[x(t)]
$$

Let $\|\cdot\|_{J}$ denote the supremum norm on $J$, i.e.,

$$
\|x(t)\|_{J}=\sup _{t \in J}\|x(t)\|, \quad x(t) \in \mathcal{C}\left(J, \mathbb{R}^{5}\right) .
$$

Then $\mathcal{C}\left(J, \mathbb{R}^{5}\right)$ with $\|\cdot\|_{J}$ is a Banach space. Moreover, it is easily shown that

$$
\left\|\int_{0}^{t} K(t, \xi) x(\xi) d \xi\right\|_{J} \leq T\|K(t, \xi)\|_{J}\|x(t)\|_{J}
$$

where $x(t) \in \mathcal{C}\left(J, \mathbb{R}^{5}\right), K(t, \xi) \in \mathcal{C}\left(J^{2}, \mathbb{R}\right)$ and

$$
\|K(t, \xi)\|_{J}=\sup _{t, \xi \in J}|K(t, \xi)|, \quad K(t, \xi) \in \mathcal{C}\left(J^{2}, \mathbb{R}\right) .
$$


Using the definition of operator $F$ in Eq. (29), and with the aid of Eqs. (16) and (32), we have

$$
\begin{aligned}
\|F[x(t)]-F[y(t)]\|_{J} \leq & \frac{1-\alpha}{B(\alpha)}\|f(x(t))-f(y(t))\|_{J} \\
& +\frac{\alpha}{B(\alpha) \Gamma(\alpha)} T^{\alpha}\|f(x(\xi))-f(y(\xi))\|_{J} \\
\leq & \left(\frac{1-\alpha}{B(\alpha)} M+\frac{\alpha}{B(\alpha) \Gamma(\alpha)} M T^{\alpha}\right)\|x(t)-y(t)\|_{J} .
\end{aligned}
$$

Therefore, we obtain

$$
\|F[x(t)]-F[y(t)]\|_{J} \leq L\|x(t)-y(t)\|_{J}
$$

where $L=\frac{1-\alpha}{B(\alpha)} M+\frac{\alpha}{B(\alpha) \Gamma(\alpha)} M T^{\alpha}$. If condition (17) is satisfied, the operator $F$ will be a contraction on $\mathcal{C}\left(J, \mathbb{R}^{5}\right)$. Thus, as a consequence of Banach fixed point theorem, system (14) has a unique solution.

\author{
Acknowledgements \\ Not applicable. \\ Funding \\ Not applicable.

\section{Competing interests} \\ The authors declare that they have no competing interests.
}

\title{
Authors' contributions
}

All authors, DB, AJ, EB, and MH, contributed equally to each part of this work. All authors read and approved the final manuscript.

\section{Author details}

${ }^{1}$ Department of Mathematics, Faculty of Arts and Sciences, Cankaya University, Ankara, Turkey. ${ }^{2}$ Institute of Space Sciences, Magurele-Bucharest, Romania. ${ }^{3}$ Department of Electrical Engineering, University of Bojnord, Bojnord, Iran. ${ }^{4}$ Department of Information Technology Education, University of Education Winneba (Kumasi Campus), Kumasi, Ghana.

${ }^{5}$ Department of Mathematics, Sahand University of Technology, Tabriz, Iran.

\section{Publisher's Note}

Springer Nature remains neutral with regard to jurisdictional claims in published maps and institutional affiliations.

Received: 16 March 2018 Accepted: 16 June 2018 Published online: 03 July 2018

\section{References}

1. Padhee, M., Zhang, S., Lie, S., Wang, K.C., Botting, K.J., McMillen, I.C., MacLaughlin, S.M., Morrison, J.L.: The periconceptional environment and cardiovascular disease: does in vitro embryo culture and transfer influence cardiovascular development and health? Nutrients 7(3), 1378-1425 (2015)

2. Cuervo, M., Sayon-Orea, C., Santiago, S., Martinez, J.A.: Dietary and health profiles of Spanish women in preconception, pregnancy and lactation. Nutrients 6(10), 4434-4451 (2014)

3. Neugebauer, R., Hoek, H.W., Susser, E.: Prenatal exposure to wartime famine and development of antisocial personality disorder in early adulthood. JAMA 282(5), 455-462 (1999)

4. Vonnahme, K.A., Lemley, C.O., Caton, J.S., Meyer, A.M.: Impacts of maternal nutrition on vascularity of nutrient transferring tissues during gestation and lactation. Nutrients 7(5), 3497-3523 (2015)

5. Zheng, J., Xiao, X., Zhang, Q., Mao, L., Yu, M., Xu, J.: The placental microbiome varies in association with low birth weight in full-term neonates. Nutrients 7(8), 6924-6937 (2015)

6. Zohdi, V., Lim, K., Pearson, J.T., Black, M.J.: Developmental programming of cardiovascular disease following intrauterine growth restriction: findings utilising a rat model of maternal protein restriction. Nutrients 7(1), 119-152 (2015)

7. Roseboom, T.J., van der Meulen, J.H., Ravelli, A.C., Osmond, C., Barker, D.J., Bleker, O.P.: Effects of prenatal exposure to the Dutch famine on adult disease in later life: an overview. Mol. Cell. Endocrinol. 185(1-2), 93-98 (2001)

8. Gitau, R., Makasa, M., Kasonka, L., Sinkala, M., Chintu, C., Tomkins, A., Filteau, S.: Maternal micronutrient status and decreased growth of Zambian infants born during and after the maize price increases resulting from the southern African drought of 2001-2002. Public Health Nutr. 8(7), 837-843 (2005) 
9. Blumfield, M.L., Nowson, C., Hure, A.J., Smith, R., Simpson, S.J., Raubenheimer, D., MacDonald-Wicks, L., Collins, C.E. Lower protein-to-carbohydrate ratio in maternal diet is associated with higher childhood systolic blood pressure up to age four years. Nutrients 7(5), 3078-3093 (2015)

10. Block, S.A., Kiess, L., Webb, P., Kosen, S., Moench-Pfanner, R., Bloem, M.W., Timmer, C.P.: Macro shocks and micro outcomes: child nutrition during Indonesia's crisis. Econ. Hum. Biol. 2(1), 21-44 (2004)

11. Colon-Ramos, U., Racette, S.B., Ganiban, J., Nguyen, T.G., Kocak, M., Carroll, K.N., Völgyi, E., Tylavsky, F.A.: Association between dietary patterns during pregnancy and birth size measures in a diverse population in southern US. Nutrients 7(2), 1318-1332 (2015)

12. Tsuduki, T., Yamamoto, K., Shuang, E., Hatakeyama, Y., Sakamoto, Y.: High dietary fat intake during lactation promotes the development of social stress-induced obesity in the offspring of mice. Nutrients 7(7), 5916-5932 (2015)

13. Martin-Prevel, Y., Delpeuch, F., Traissac, P., Massamba, J.P., Adou-Oyila, G., Coudert, K., Treche, S.: Deterioration in the nutritional status of young children and their mothers in Brazzaville, Congo following the 1994 devaluation of the CFA franc. Bull. World Health Organ. 78(1), 108-118 (2000)

14. Rao, S., Yajnik, C.S., Kanade, A., Fall, C.H., Margetts, B.M., Jackson, A.A., Shier, R., Joshi, S., Rege, S., Lubree, H., Desai, B.: Intake of micronutrient-rich foods in rural Indian mothers is associated with the size of their babies at birth: Pune Maternal Nutrition Study. J. Nutr. 131(4), 1217-1224 (2001)

15. Silva, C.J., Torres, D.F.M.: A SICA compartmental model in epidemiology with application to HIV/AIDS in Cape Verde. Ecol. Complex. 30, 70-75 (2017)

16. Wang, Y., Liu, J., Liu, L.: Viral dynamics of an HIV model with latent infection incorporating antiretroviral therapy. Adv Differ. Equ. 2016, Article ID 225 (2016)

17. Kiryakova, V.S.: Generalized Fractional Calculus and Applications. CRC Press, Boca Raton (1993)

18. Liu, Z., Lu, P.: Stability analysis for HIV infection of CD4+ T-cells by a fractional differential time-delay model with cure rate. Adv. Differ. Equ. 2014, Article ID 298 (2014)

19. Bolton, L., Cloot, A.H., Schoombie, S.W., Slabbert, J.P.: A proposed fractional-order Gompertz model and its application to tumour growth data. Math. Med. Biol. 32(2), 187-207 (2015)

20. Bodo, B., Mvogo, A., Morfu, S.: Fractional dynamical behavior of electrical activity in a model of pancreatic $\beta$-cells. Chaos Solitons Fractals 102, 426-432 (2017)

21. Arshad, S., Baleanu, D., Bu, W., Tang, Y.: Effects of HIV infection on CD4+ T-cell population based on a fractional-order model. Adv. Differ. Equ. 2017, Article ID 92 (2017)

22. Ford, N.J., Simpson, A.C.: The numerical solution of fractional differential equations: speed versus accuracy. Numer. Algorithms 26(4), 333-346 (2001)

23. Toufik, M., Atangana, A.: New numerical approximation of fractional derivative with non-local and non-singular kernel: application to chaotic models. Eur. Phys. J. Plus 132, Article ID 444 (2017)

24. Atangana, A., Jain, S.: A new numerical approximation of the fractal ordinary differential equation. Eur. Phys. J. Plus 133, Article ID 37 (2018)

25. Owolabi, K.M., Atangana, A.: Analysis and application of new fractional Adams-Bashforth scheme with Caputo-Fabrizio derivative. Chaos Solitons Fractals 105, 111-119 (2017)

26. Bonyah, E., Atangana, A., Khan, M.A.: Modeling the spread of computer virus via Caputo fractional derivative and the beta-derivative. Asia Pac. J. Comput. Eng. 4, Article ID 1 (2017)

27. Arshad, S., Baleanu, D., Huang, J., Tang, Y., Al Qurashi, M.M.: Dynamical analysis of fractional order model of immunogenic tumors. Adv. Mech. Eng. 8(7), 1-13 (2016)

28. Alkahtani, B.S.T., Atangana, A., Koca, I.: Huge analysis of Hepatitis $\mathrm{C}$ model within the scope of fractional calculus. J. Nonlinear Sci. Appl. 9, 6195-6203 (2016)

29. Pinto, C.M.A., Machado, J.A.T.: Fractional model for malaria transmission under control strategies. Comput. Math. Appl. 66(5), 908-916 (2013)

30. Pinto, C.M.A., Carvalho, A.R.M.: Fractional complex-order model for HIV infection with drug resistance during therapy. J. Vib. Control 22(9), 2222-2239 (2016)

31. Huo, J., Zhao, H., Zhu, L.: The effect of vaccines on backward bifurcation in a fractional order HIV model. Nonlinear Anal., Real World Appl. 26, 289-305 (2015)

32. Rostamy, D., Mottaghi, E.: Forward and backward bifurcation in a fractional-order SIR epidemic model with vaccination. Iran. J. Sci. Technol. Trans. A, Sci. (2018). https://doi.org/10.1007/s40995-018-0519-7

33. Rostamy, D., Mottaghi, E.: Stability analysis of a fractional-order epidemics model with multiple equilibriums. Adv. Differ. Equ. 2016, Article ID 170 (2016)

34. Rostamy, D., Mottaghi, E.: Numerical solution and stability analysis of a nonlinear vaccination model with historical effects. Hacet. J. Math. Stat. (2017). https://doi.org/10.15672/HJMS.20174720333

35. Atangana, A., Baleanu, D.: New fractional derivatives with nonlocal and non-singular kernel: theory and application to heat transfer model. Therm. Sci. 20(2), 763-769 (2016)

36. Podlubny, I.: Fractional Differential Equations: An Introduction to Fractional Derivatives, Fractional Differential Equations, to Methods of Their Solution and Some of Their Applications. Academic Press, New York (1999)

37. Kilbas, A.A., Srivastava, H.H., Trujillo, J.J.: Theory and Applications of Fractional Differential Equations. Elsevier, New York (2006)

38. Hilfer, R.: Applications of Fractional Calculus in Physics. World Scientific, Singapore (2000)

39. Wu, G.C., Baleanu, D., Zeng, S.D., Deng, Z.G.: Discrete fractional diffusion equation. Nonlinear Dyn. 80, 281-286 (2015)

40. Atangana, A.: On the new fractional derivative and application to nonlinear Fisher's reaction-diffusion equation. Appl. Math. Comput. 273, 948-956 (2016)

41. Coronel-Escamilla, A., Aguilar, J.F., Baleanu, D., Escobar-Jimenez, R.F., Olivares-Peregrino, V.H., Abundez-Pliego, A.: Formulation of Euler-Lagrange and Hamilton equations involving fractional operators with regular kernel. Adv. Differ. Equ. 2016, Article ID 283 (2016)

42. Atangana, A., Koca, l.: Chaos in a simple nonlinear system with Atangana-Baleanu derivatives with fractional order. Chaos Solitons Fractals 89, 447-454 (2016)

43. Abdeljawad, T., Baleanu, D.: Discrete fractional differences with nonsingular discrete Mittag-Leffler kernels. Adv. Differ. Equ. 2016, Article ID 232 (2016) 
44. Shah, N.H., Thakkar, F.A., Yeolekar, B.M.: Mathematical analysis of optimal control theory on underweight. Adv. Res. 8(5), Article ID AIR.30992 (2016)

45. Al-Sulami, H., El-Shahed, M., Nieto, J.J., Shammakh, W.: On fractional order dengue epidemic model. Math. Probl. Eng. 2014, Article ID 456537 (2014)

46. Hethcote, H., Zhien, M., Shengbing, L.: Effects of quarantine in six endemic models for infectious diseases. Math. Biosci. 180(1-2), 141-160 (2002)

47. van den Driessche, P., Watmough, J.: Reproduction numbers and sub-threshold endemic equilibria for compartmental models of disease transmission. Math. Biosci. 180(1-2), 29-48 (2002)

Submit your manuscript to a SpringerOpen ${ }^{\circ}$ journal and benefit from:

- Convenient online submission

- Rigorous peer review

- Open access: articles freely available online

- High visibility within the field

- Retaining the copyright to your article

Submit your next manuscript at $\gg$ springeropen.com 